\title{
Potential of multispectral imaging technology for assessment coniferous forests bitten by a bark beetle in Central Bulgaria
}

\author{
Marina Stoyanova ${ }^{1}$, Alexander Kandilarov ${ }^{2}$, Vesselin $\mathrm{Koutev}^{3}$, Olga Nitcheva $^{4,}{ }^{*}$, Polya \\ Dobreva $^{4}$ \\ ${ }^{1}$ Agriculture Institute, Industrialna Str. 1, 8400 Karnobat, Bulgaria \\ ${ }^{2}$ Neopterix Ltd, 4000 Plovdiv, Bulgaria \\ ${ }^{3}$ University of Forestry, Kliment Ohridski Str. 10, 1756, Sofia, Bulgaria \\ ${ }^{4}$ Institute of Mechanics - BAS, Acad. G. Bonchev Str., Bl. 4, 1113, Sofia, Bulgaria
}

\begin{abstract}
Much of the coniferous forest in Bulgaria is struck by Ips typographus, Ips acuminatus and Ips sexdentatus beetles. The multispectral camera test near the town of Kalofer, Bulgaria in August 2017, showed the good capabilities of remote-piloted aircraft for observation and mapping of large areas in highly intersected areas. The collected data from the multispectral camera allowed producing maps with different indices of the observed vegetation. Beetles damaged stains were identified and outlining the areas of intervention and their sizing is possible by forestry specialists. The NDVI index in the range of 0.7 to 0.95 characterizes green tree vegetation. Affected forest subjected to stress has lower NDVI index values - 0.65.The NDVI index of damaged areas range from 0.45-0.5. Identifying affected forest areas is the first important step to solve the problem. Taking pictures of drones and multispectral camera can be of great help, and the test conducted clearly demonstrates it. Preventive actions can happen much earlier before trees are completely destroyed and the problem is clearly visible with the naked eye. In view of the rapid spread and the complete destruction of some conifers, immediate action is needed to cut out and remove the diseased and infected trees in order to break the development cycle and prevent subsequent contamination of adjacent plots
\end{abstract}

\section{Introduction}

The protection of Bulgarian forests is a priority given in the National Strategy for Development of the Forestry Sector in the Republic of Bulgaria for the period 2013-2020. Forests fulfill many economic, ecological and social functions of particular importance for the sustainable development of the country and are a key factor for the formation and maintenance of a living environment. Much of the coniferous forest is struck by Ips typographus beetles, Ips acuminatus, Ips sexdentatus. This requires urgent measures to

\footnotetext{
*Corresponding author: olgani@imbm.bas.bg
} 
clean up the damaged terrain and prevent further contamination. In the opinion of Eng. Grigor Gogov (Director of the Executive Agency of Forests), the main reason for the increase of the bark beetles attacks is the good nutritional environment for their development. It is believed that the large amount of wood - over 2 million cubic meters damaged by abiotic factors - winds, windmills, ice and snow - combined with the impossibility of fast and removal within one year due to the high percentage of inaccessible terrains, are factors that require adequate measures to save the forest.

The only way to deal with the problem is to cut out the damaged trees and immediately remove them from the terrain. Given the percentage of diseased trees and the area of the affected area, the situation can be assessed as disastrous. To deal with this situation serious organizational, human and financial resources are required. In such situations, however, the first step is to carry out quality monitoring to accurately quantify the problem. Till now monitoring of bark beetles was assessed by observation of affected areas or application of pheromone traps [1]. The analysis of damages caused by bark beetle on Norway spruce (Picea abies) forest in Vitosha mountain, Bulgaria was applied using the VIs indicative for forest stress: NDVI, SR, EVI, ARVI, CRI, CSc, and ARI. Main regions for stressed vegetation have been delineated. The CSc has been found to perform better in detecting the pattern of stressed spruce trees compared to ARI. The area affected by Ips typographus was determined by CSc index to $5.97 \%\left(0.373 \mathrm{~km}^{2}\right)$ of the study area [2]. With the aid of the object-oriented image analysis software eCognition classification in the deadwood areas damaged by bark beetle yielded a total classification accuracy of $91.5 \%$ [3].

Taking into account the wide spread of bark beetles spots on hard-to-reach forest areas, remote monitoring methods are the most effective way of conducting observations, sizing the affected area, assessing the risk and planning the necessary resources for follow-up. Remote sensing techniques are used to generate maps and assess the different degrees of attack. Spectral and geometric methods of high-resolution remote monitoring serve to characterize the health or vitality of trees. Stress in vegetation leads to changes in the composition and proportion of photosynthetic active pigments such as chlorophyll a and $b$ as well as in plant foliage. This is also the reason why changes in the health status of the forest can be observed at a very early stage [4].

\section{Materials and methods}

Bark beetles are small beetles, which, depending on the species, range from 2.5 to $8 \mathrm{~mm}$ (Figure 1). They develop two generations per year, and are frequent below $800 \mathrm{~m}$ above sea level. In the warmer years it is possible to have three generations. The first generation flies in early May, and the second in July and the beginning of August. They feed with coniferous wood, mostly white pine and spruce.

Of the representatives of the bark beetles in Bulgaria, the prevalence and damage of Ips acuminatus is greatest [5 - 6]. It primarily affects white pine (Pinus sylvestris L.). Its color is yellow-brown, the larvae is $6 \mathrm{~mm}$ long, white and without legs. Propagation is under the bark of the top of the stems and the thick branches. The male is digging a wedding chamber for the females. Each female makes a motherly move in which they lay eggs. The course of the larvae is short and ends with a cocoon chamber. They develop sister generations. It attacks the top of the stems with a smooth bark. Mothers and lashes move the conductive tissue of the attacked tree and it quickly dries out Figure 2. 


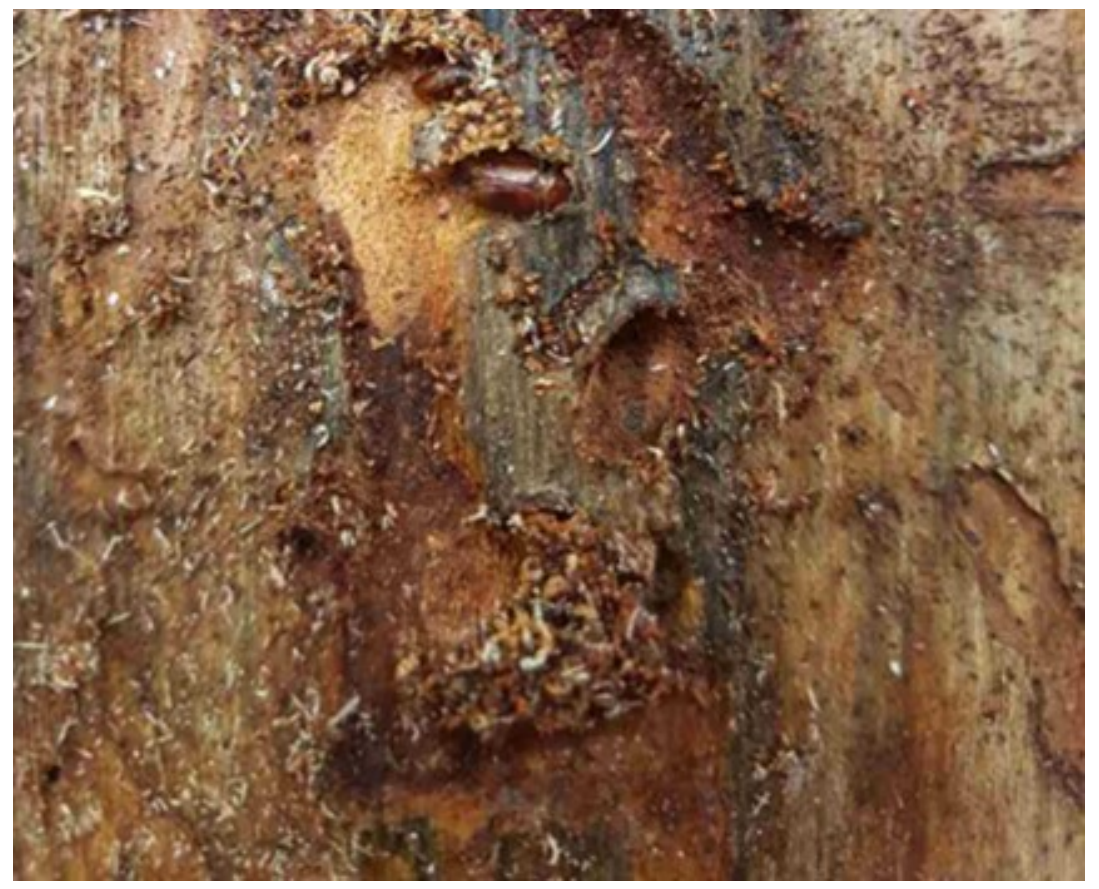

Fig. 1. Bark beetle under the removed bark.

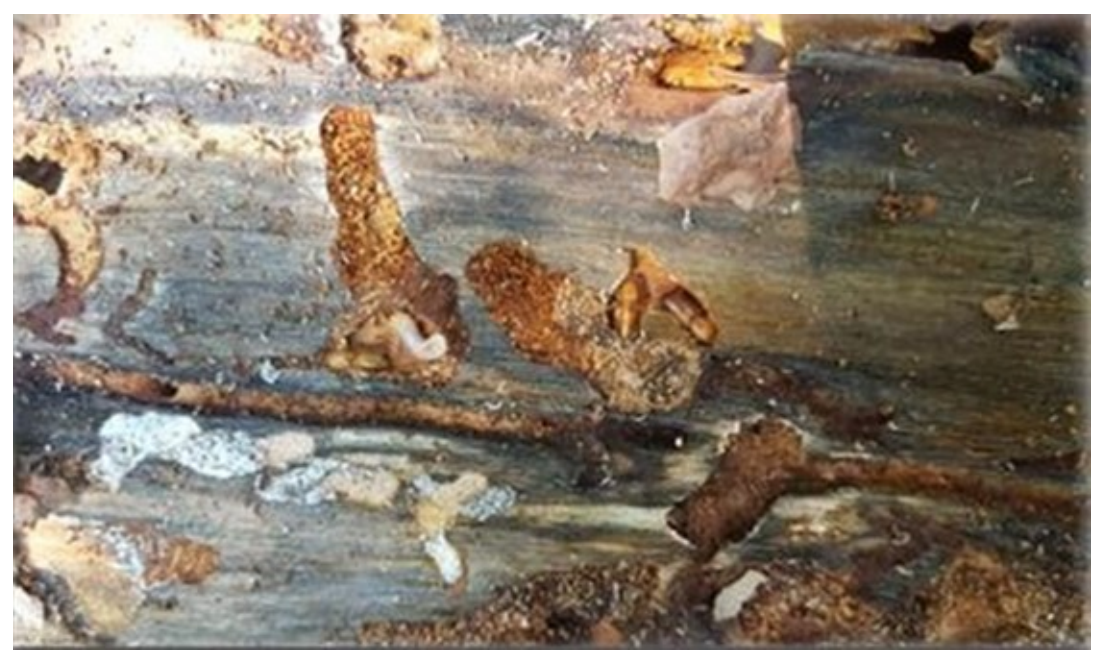

Fig. 2. Bark beetle damages.

Although bark beetles tend to colonize dead or weakened trees, there are reports that some species attack healthy trees under epidemic conditions, attacking individual trees or groups of trees, resulting in so-called bark beetle spots - Figure 3. 


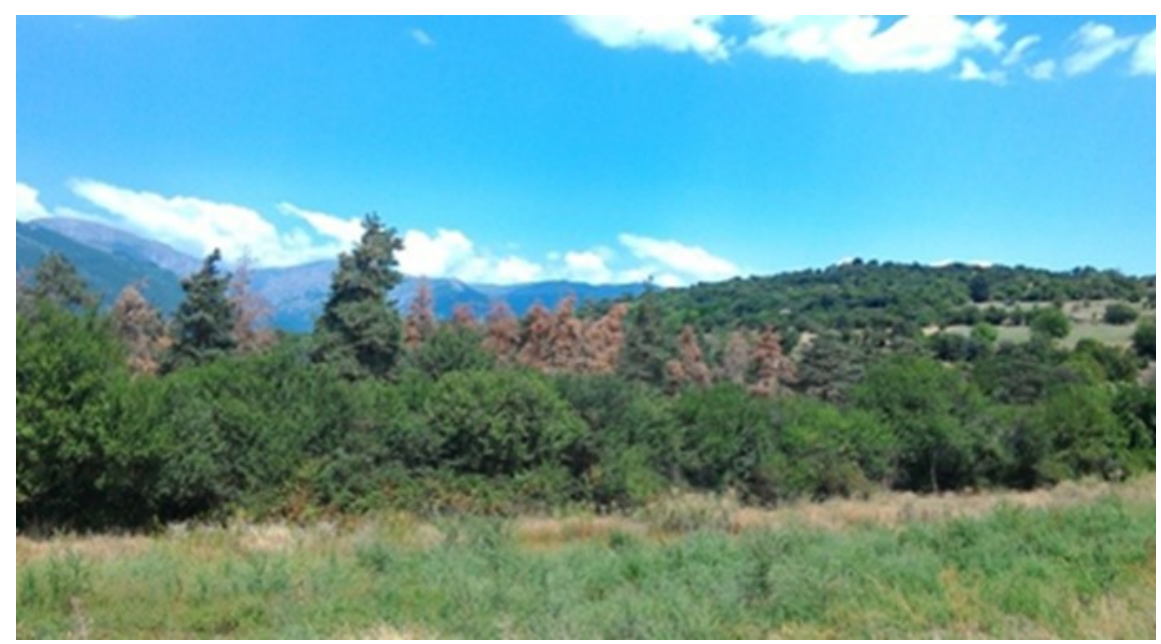

Fig. 3. Bark beetle spots.

Each type of tree has different reflectivity across the different regions of the electromagnetic spectrum. It can be seen from Figure 4 that the reflectivity of deciduous forests varies in a much larger range at the end of the visible portion of the electromagnetic spectrum (beyond the red end of the spectrum and in the near-infrared wavelength of 700 $\mathrm{nm}$ and greater). This can be used to easily distinguish between the two types of vegetation in mixed forests. Other specificity of NDVI indexes from different surfaces are shown on Figures 5 and 6.

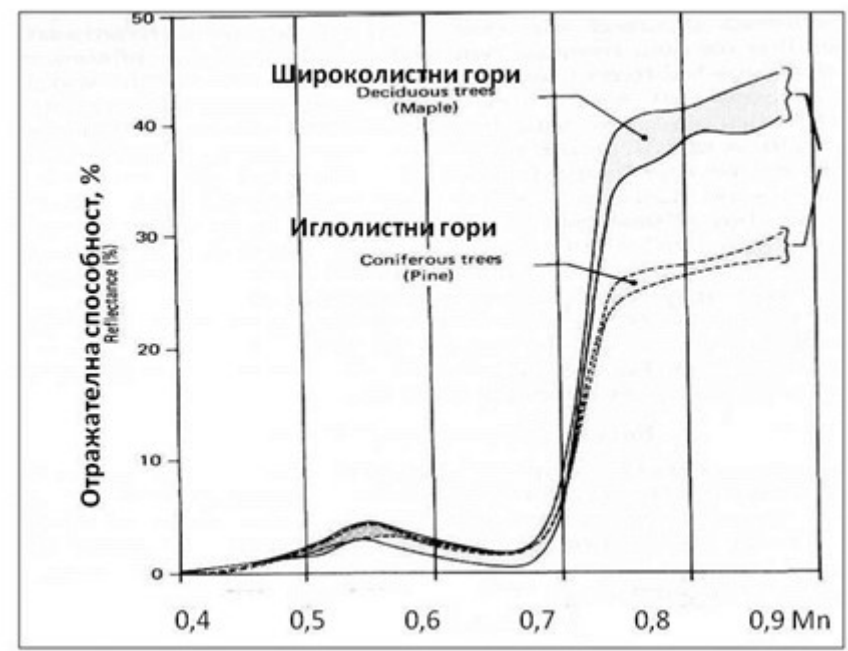

Fig. 4. Difference in the reflectance of deciduous and coniferous trees [7]. 


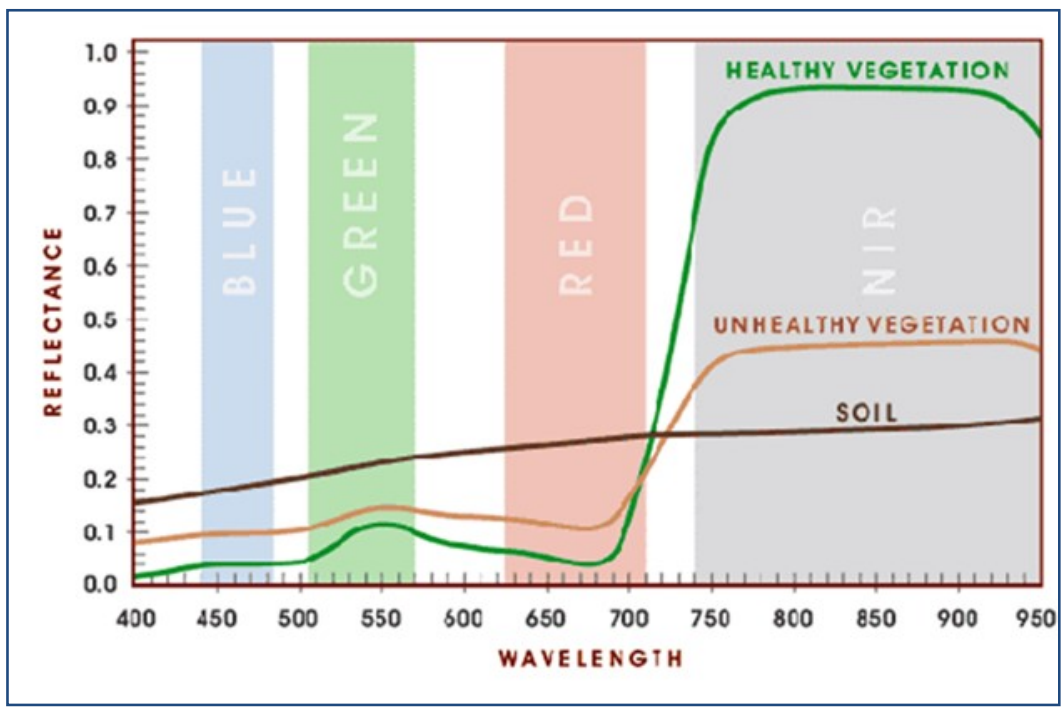

Fig. 5. Combined reflectivity of soil and vegetation - note the difference in the near infrared range between the healthy and diseased vegetation curves [8].

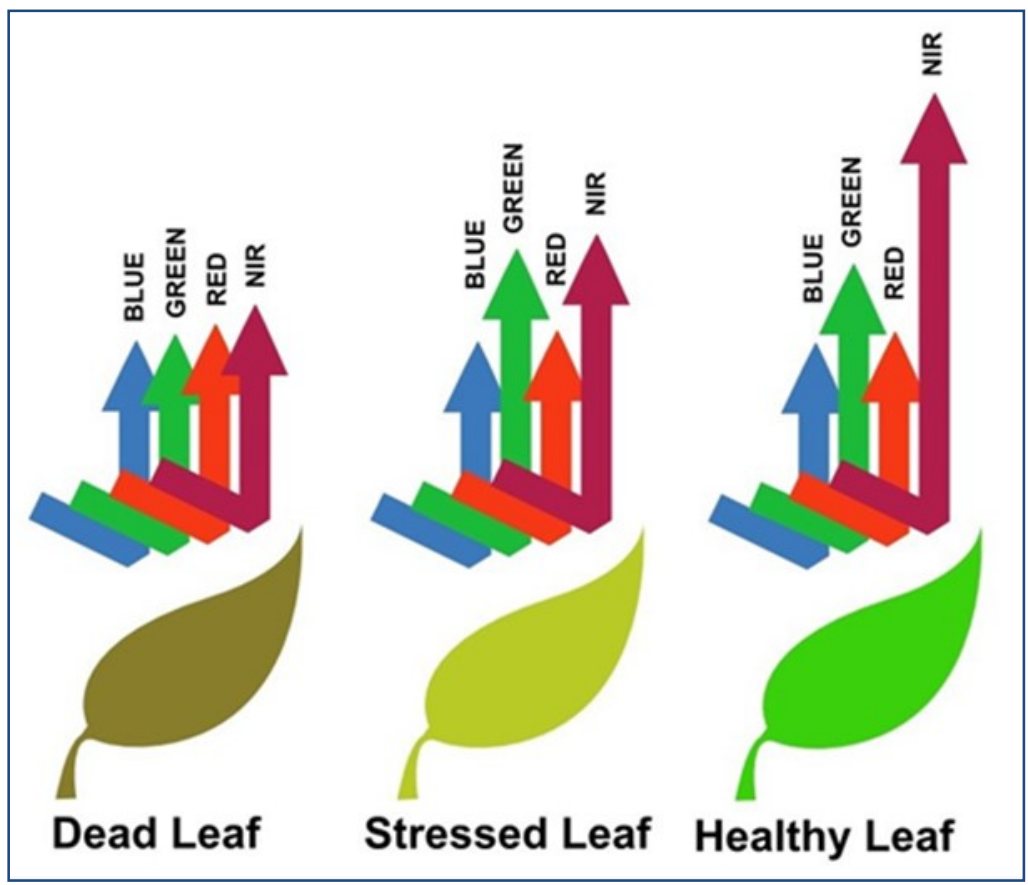

Fig. 6. Schematic presentation of the reflection ability of dead, stressed and healthy vegetation [9].

Healthy plants generally have a higher reflectance in the near infrared area than diseased and stressed plants, which can be used to distinguish one from the other. In remote studies, the so-called NDVI (Normalized Difference Vegetation Index) which is a numeric indicator using the visible (RED) and near infrared areas (NIR) of the electromagnetic spectrum for the analysis of vegetation and vegetation strength. Typically, healthy vegetation takes up much of the visible light that falls on it, and reflects much of the nearby 
infrared area. Damaged or rare vegetation reflects more than visible light and less than the near infrared.

$$
\mathrm{NDVI}=(\mathrm{NIR}-\mathrm{RED}) /(\mathrm{NIR}+\mathrm{RED})
$$

The study used one aircraft of Neopteryx Ltd. with identification number NTRX002. The NTRX002 is the DJI Matrix 600 Pro Hexcopter. It is equipped with a 5-channel multispectral camera, consisting of 16 megapixel standard optical sensor (RGB) and 4 separate sensors capturing light in green, red, extreme red and near infrared of the electromagnetic spectrum. It is additionally equipped with a 16 megapixel optical camera. Its weight in configuration with the two cameras is $12 \mathrm{~kg}$, its radius of action is $4.5 \mathrm{~km}$ and the dynamic flight ceiling is $4500 \mathrm{~m}$.

The resulting raw RGB camera results are in JPEG format, and from the multi-spectral camera - in a georeferenced TIFF format.

The survey was accomplished hear the city of Kalofer in Central Bulgaria, Figure 7.

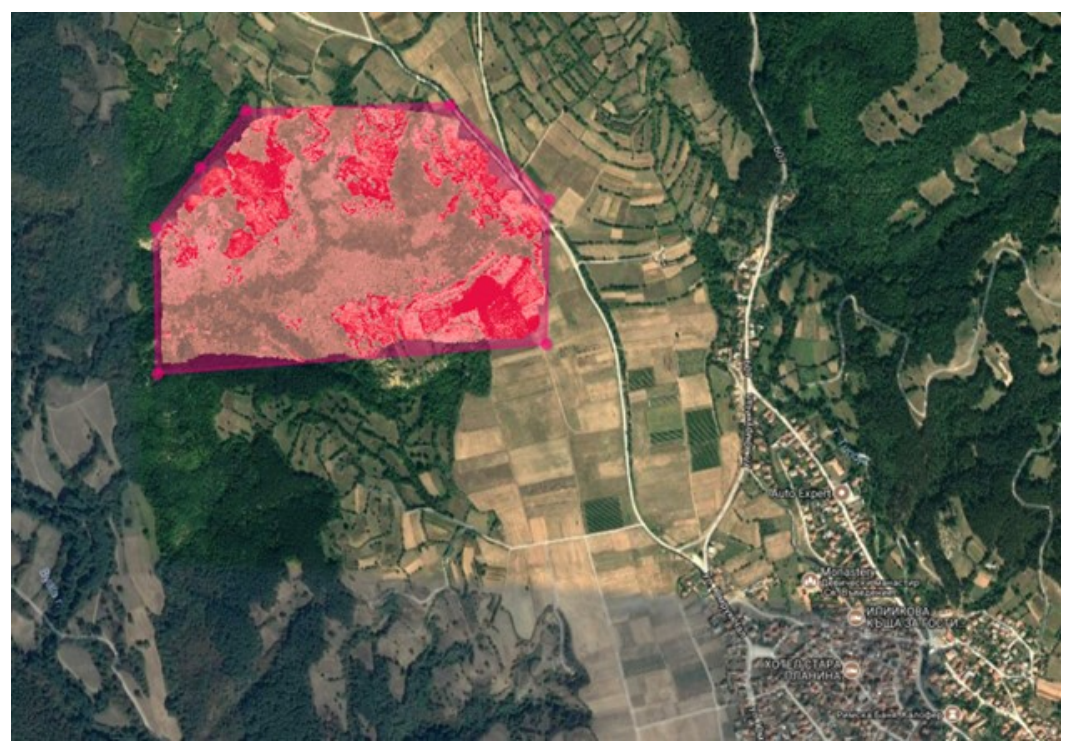

Fig. 7. Satellite image of the surveyed area. ( Google Earth Satellite Imagery).

Objectives of the study are to capture damaged forest area with a drone equipped with a five-strip multi-spectral camera and an additional optical camera and to generate maps with different vegetation indices.

\section{Results}

The results of the field survey are images from the multispectral camera. After processing digital maps are obtained in different bands of the electromagnetic spectrum.

The whole area studied, with the exception of the access roads and the access to it, is a mixed forest, which was clearly visible at the time of the study, and several damaged areas were observed.

To separate the deciduous vegetation, a reflection capacity map is used at the far red end of the visible portion of the electromagnetic spectrum, and the vegetation classification by height and slope of the 3D cloud from points (Figure 8). 


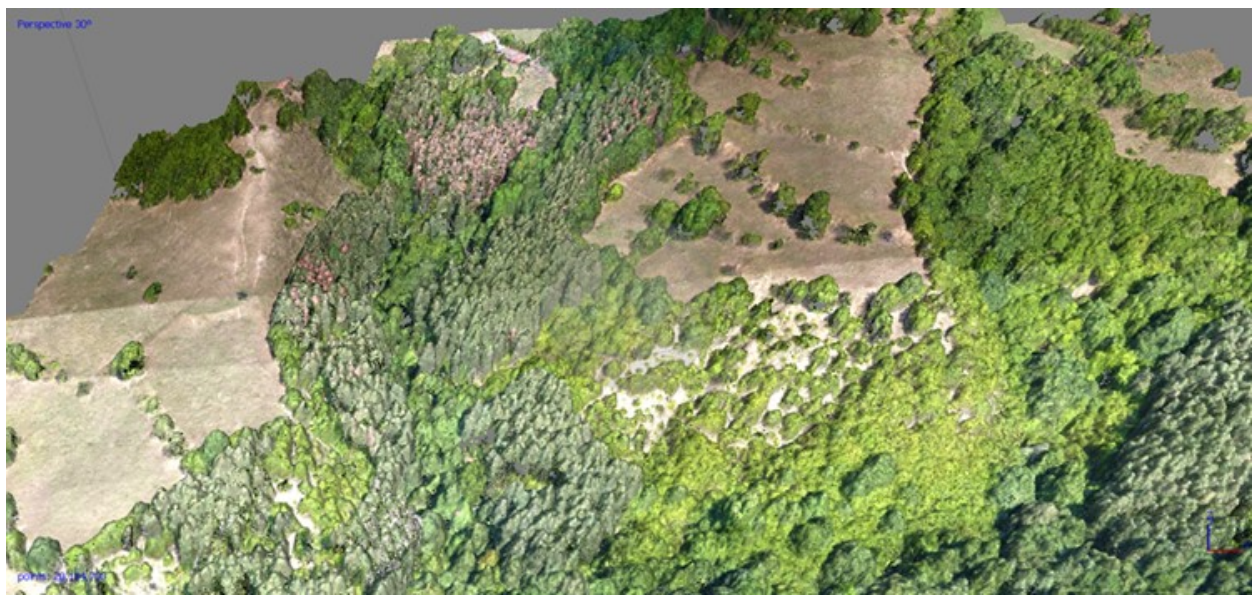

Fig. 8. Part of a 3D model of the terrain, showing a bark beetle spot in the northern part.

A map of the NDVI index (Formula 1) is used to assess the strength of forest vegetation. Photos and maps of the northern and southern parts of the surveyed area are given in Figures 9, 10, 11, 12. For analysis, these two parts of the site are selected because they contain all the elements of the problem that the test to decide - this is the presence of mixed deciduous and coniferous forest vegetation, damaged by bark beetle coniferous trees and damaged spots, the presence of pastures, meadows, arable land and low buildings, which are hindering factors in the analysis of altitude data.

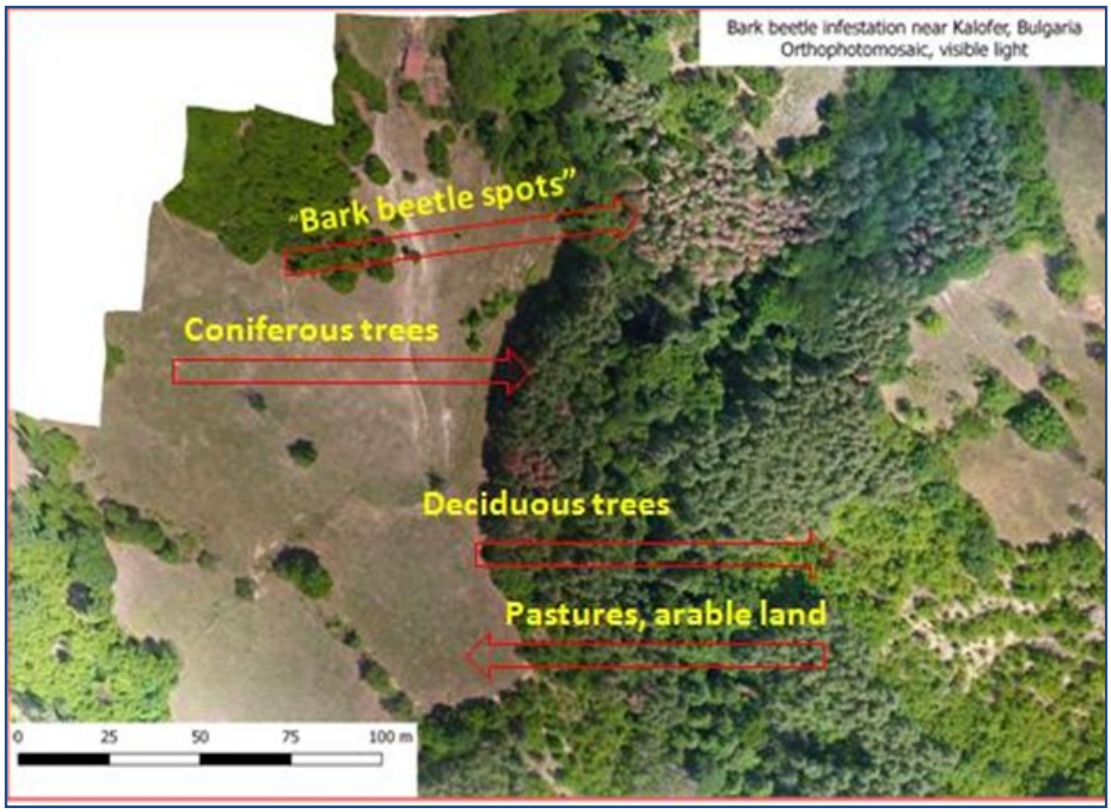

Fig. 9. Part of the orthophotomap at the northern end of the surveyed forest massif. 


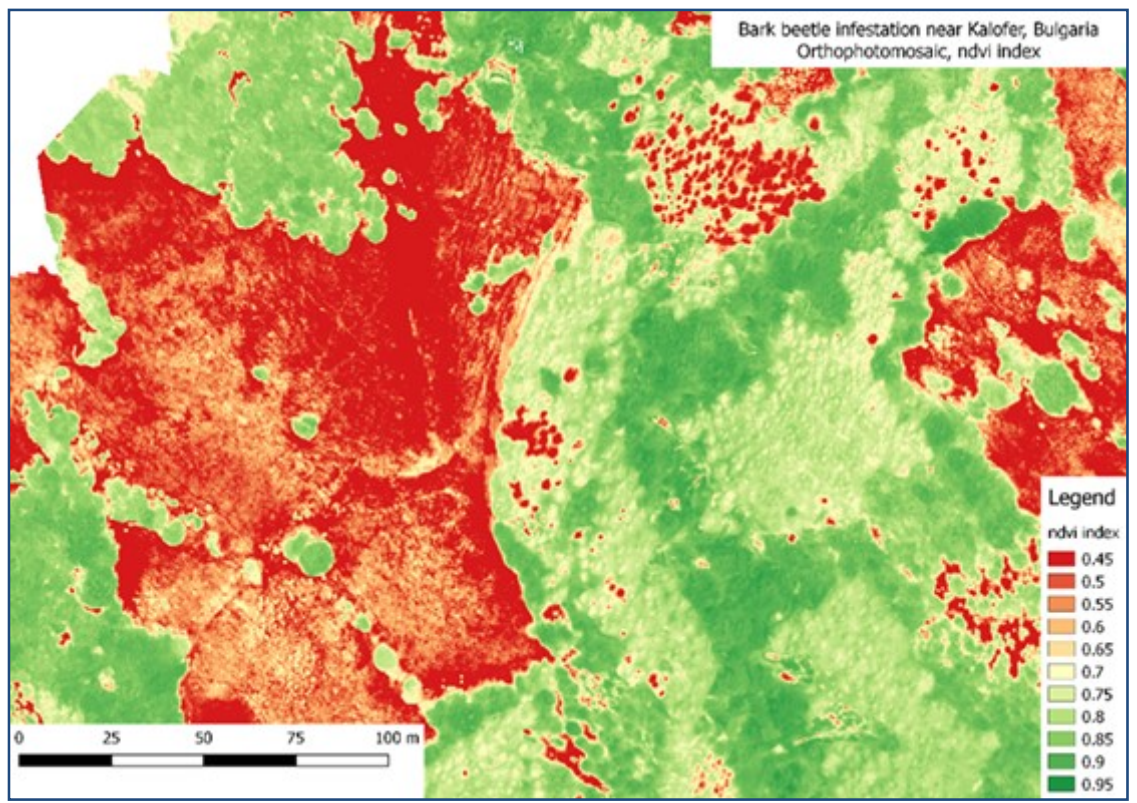

Fig. 10. Map of the NDVI index in the northern part of the surveyed terrain.

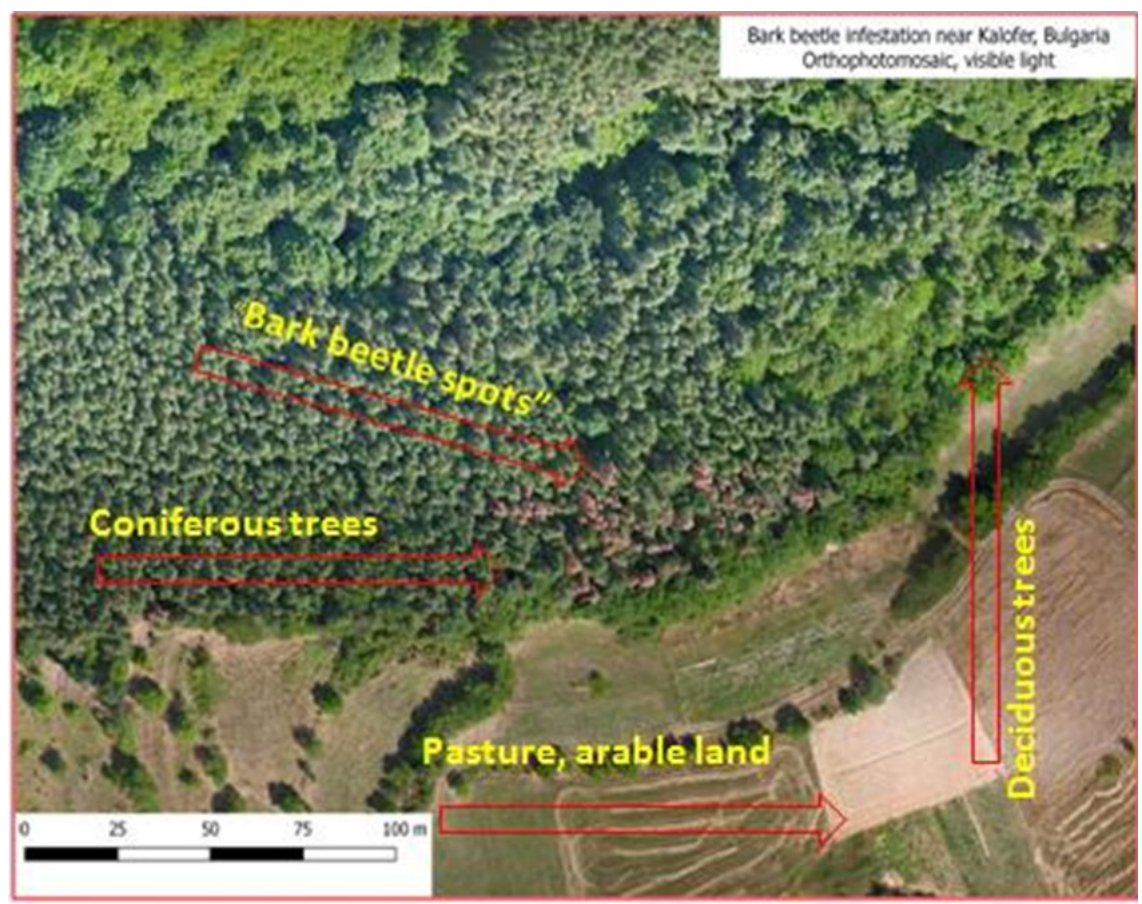

Fig. 11. Part of the orthophotomap at the southern end of the investigated forest. 


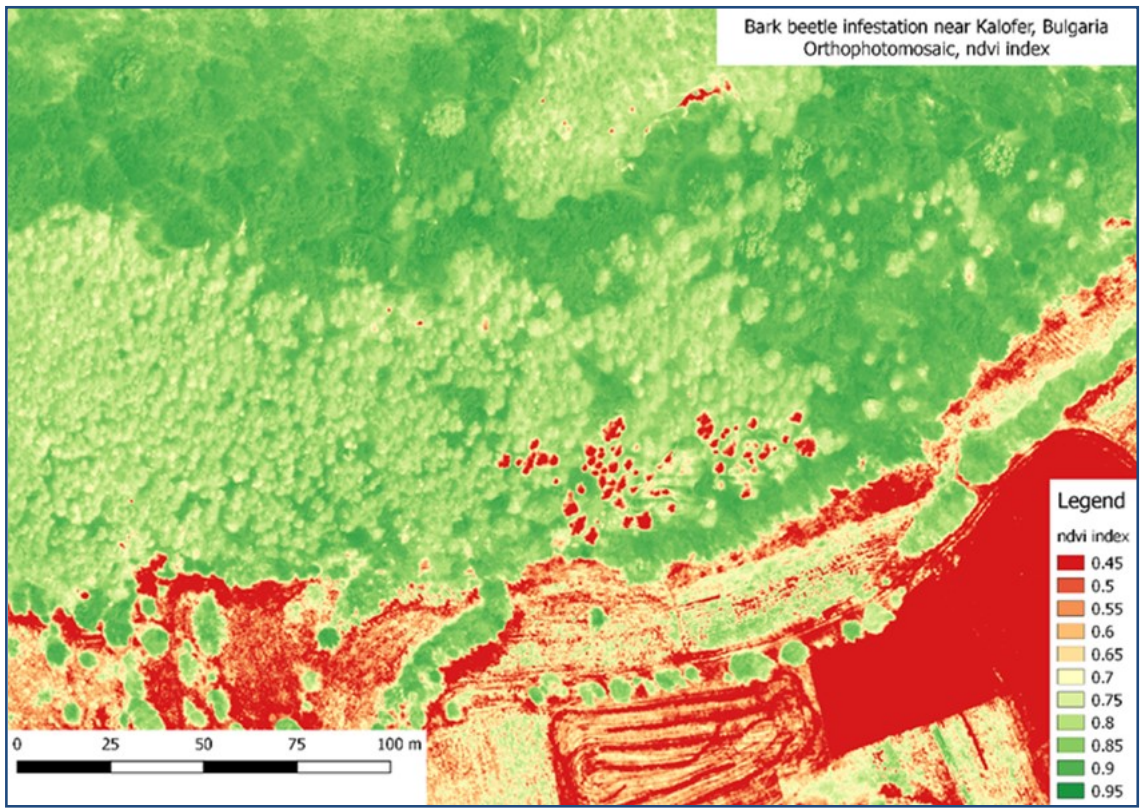

Fig. 12. Map of the NDVI index in the southern part of the surveyed terrain.

The various indexes generated by the multispectral camera data together with the $3 \mathrm{D}$ cloud of points help to distinguish deciduous forests from coniferous forests and subsequently identify bark beetle spots and areas of varying degrees of damage from bark beetles and other pests. Quantitative dimensioning of the affected area can be done in the Geographic Information Systems (GIS) and, after consultation with forestry specialists the intervention areas can be outlined.

In the middle of August 2017, when the study was conducted, the vegetation development phase was such that the NDVI field index was below or around 0.45-0.5 (red in the corresponding figures). The NDVI index in the range of 0.7 to 0.95 characterizes green tree vegetation. On both sides of the coniferous forest are fields in clear red color (Figures 9 and 10). In the middle of the terrain are coniferous trees, the spots from red to yellow outline the bark beetle affected trees. The NDVI index score is estimated to range from 0.45-0.5. Spots in red on coniferous stretches outline areas with affected or completely destroyed trees - a deep red color is a sign of it - these are the affected spots.

Similar is the situation in the south. In the lower part of the studied area there are fields and pastures (NDVI 0.45-0.55). Yellow and green is forest vegetation, with a healthy NDVI index of between 0.85 and 0.95 . The forest that has been subjected to stress has less reflectivity in the red and near infrared, resulting in lower NDVI index values, and it drops to 0.65 . Damaged spots are slightly above 0.5 , as a result of which they are easily distinguishable.

\section{Achieved goals}

The multispectral camera test near the town of Kalofer showed the good capabilities of remote-piloted aircraft for observation and mapping of large areas in highly impassable terrain. Performing such a study on the same area at the same time by foot across the terrain would be impossible. 
The collected data from the optical camera allowed the generation of orthophotomaps and a $3 \mathrm{D}$ model of the terrain, and data from the multispectral camera allowed to produce maps with different indices of the observed vegetation.

A problem with mixed forests is the separation of areas with deciduous trees from those with coniferous vegetation. In this test, this problem was solved in two steps - by classifying the $3 \mathrm{D}$ cloud by points at relative altitudes and gradients, and by classifying the areas in the reflectance map at the red end of the visible portion of the electromagnetic spectrum.

\section{Conclusion}

The forest vegetation in the studied terrain is subject to bark beetle stress. Identifying affected forest areas is the first important step to solve the problem. Taking pictures of drones and multispectral camera can be of great help, and the test carried out clearly demonstrates it. The elevation data from it shows that, in addition to bark beetle damaged spots that can be correctly identified this can happen much earlier than when the trees are completely destroyed and the problem is clearly visible with the naked eye. Earlier identification of problem areas will help to take timely measures and prevent the spreading of the bark beetle in adjacent areas. The study found that so far the damaged spots occupy a small part of the area surveyed. In view of the rapid spread of bark beetles and the complete destruction of some conifers, immediate action is needed to cut out and remove the diseased and infected trees in order to break the bark beetle development cycle and prevent subsequent contamination of adjacent plots.

\section{References}

1. B. Chandler, Monitoring Abundance of Ips Bark Beetles and Determining Related Tree Mortality in Arkansas and Texas (Dissertations, paper 21, 2015)

2. L. Filchev, Proceedings of 1st European SCGIS Conference with International Participation "Best Practices: Application of GIS Technologies for Conservation of Natural and Cultural Heritage Sites" (SCGIS-Bulgaria, Sofia, 2012)

3. M. Heurich, T. Ochs, T. Andresen, T. Schneider, Eur J Forest Res 129, 313 (2010).

4. A. Lausch, M. Heurich, D. Gordalla, H. Dobner, S. Margianto, C. Salback, Forest Ecology and Management 308, 76 (2013)

5. B. Rosnev, P. Mirchev, G. Georgiev, P. Petkov, Y. Naydenov, G. Tsankov, D. Ovcharov, St. Mirchev, A. Pencheva, D. Doychev, M. Matova, M. Georgieva, Handbook of forest protection. Part I. Diseases, insects and other pests and damages on forest trees and bushes (Obrazovanie i nauka, Sofia, 2006)

6. S. Lopes, A. Goldarazena, Journal of Entomology 2012, Article ID 145930 (2012)

7. T.M. Lillesand, R.W. Keiefer, J. Chipman, Remote sensing and Image Interpretation 7ed. (2015)

8. B. Warzak, Vision Systems Design, November (2016)

9. Agribotix.com, Misconceptions about UAV-collected NDVI imagery and the Agribotix experience in ground truthing these images for agriculture (2017) 